\title{
Interrater Agreement and Detection Accuracy for Medium- Vessel Occlusions Using Single-Phase and Multiphase CT Angiography
}

\author{
(D).M. Ospel, (DF. Bala, (DR.V. McDonough, (D). Volny, DN. Kashani, (DW. Qiu, (D)B.K. Menon, and (D). Goyal
}

\begin{abstract}
BACKGROUND AND PURPOSE: Accurate and reliable detection of medium-vessel occlusions is important to establish the diagnosis of acute ischemic stroke and initiate appropriate treatment with intravenous thrombolysis or endovascular thrombectomy. However, medium-vessel occlusions are often challenging to detect, especially for unexperienced readers. We aimed to evaluate the accuracy and interrater agreement of the detection of medium-vessel occlusions using single-phase and multiphase CTA.
\end{abstract}

MATERIALS AND METHODS: Single-phase and multiphase CTA of 120 patients with acute ischemic stroke (20 with no occlusion, 44 with large-vessel occlusion, and 56 with medium-vessel occlusion in the anterior and posterior circulation) were assessed by 3 readers with varying levels of experience (session 1: single-phase CTA; session 2: multiphase CTA). Interrater agreement for occlusion type (large-vessel occlusion versus medium-vessel occlusion versus no occlusion) and for detailed occlusion sites was calculated using the Fleiss $\kappa$ with $95 \%$ confidence intervals. Accuracy for the detection of medium-vessel occlusions was calculated for each reader using classification tables.

RESULTS: Interrater agreement for occlusion type was moderate for single-phase CTA $(\kappa=0.58$; $95 \% \mathrm{Cl}, 0.56-0.62)$ and almost perfect for multiphase CTA $(\kappa=0.81 ; 95 \% \mathrm{Cl}, 0.78-0.83)$. Interrater agreement for detailed occlusion sites was moderate for singlephase CTA $(\kappa=0.55 ; 95 \% \mathrm{Cl}, 0.53-0.56)$ and substantial for multiphase CTA $(\kappa=0.71 ; 95 \% \mathrm{Cl}, 0.67-0.74)$. On single-phase CTA, readers 1, 2, and 3 classified 33/56 (59\%), 34/56 (61\%), and 32/56 (57\%) correctly as medium-vessel occlusions. On multiphase CTA, $48 / 56(86 \%), 50 / 56(89 \%)$, and 50/56 (89\%) medium-vessel occlusions were classified correctly.

CONCLUSIONS: Interrater agreement for medium-vessel occlusions is moderate when using single-phase CTA and almost perfect with multiphase CTA. Detection accuracy is substantially higher with multiphase CTA compared with single-phase CTA, suggesting that multiphase CTA might be a valuable tool for assessment of medium-vessel occlusion stroke.

ABBREVIATIONS: EVT = endovascular treatment; LVO = large-vessel occlusion; mCTA = multiphase CTA; MeVO = medium-vessel occlusion

M edium-vessel occlusions (MeVOs) are defined as occlusions of the M2 and M3/4 segments of the MCA, A2 and A3/4 segments of the anterior cerebral artery, and P2 and P3/4

Received August 10, 2021; accepted after revision September 22.

From the Department of Diagnostic Imaging (J.M.O., F.B., R.V.M., N.K., W.Q., B.K.M., M.G.), and Department of Clinical Neurosciences (N.K., B.K.M., M.G.), University of Calgary, Calgary, Alberta, Canada; Department of Radiology (J.M.O.), University Hospital of Basel, Basel, Switzerland; Czech National Centre for Evidence-Based Healthcare and Knowledge Translation (O.V.), Masaryk University, Brno, Czech; and Department of Neurology (O.V.), University Hospital Ostrava, Ostrava, Czech. This study was funded, in part, by the Canadian Institutes of Health Research. Please address correspondence to Mayank Goyal, MD, Departments of Diagnostic Imaging and Clinical Neurosciences, Foothills Medical Centre, 1403 29th St NW, University of Calgary, Calgary, Alberta, Canada, T2N2T9; e-mail:

mgoyal@ucalgary.ca; @johanna_ospel; @rosevmcd; @mayank_G0

- Indicates open access to non-subscribers at www.ajnr.org

Indicates article with online supplemental data.

http://dx.doi.org/10.3174/ajnr.A7361 segments of the posterior cerebral artery. ${ }^{1} \mathrm{MeVOs}$ account for approximately $25 \%-40 \%$ of all acute ischemic strokes, and although they have traditionally been thought to cause only minor symptoms, MeVO strokes frequently result in disabling deficits. ${ }^{2,3}$ This outcome has led to a paradigm shift in treatment: An increasing number of physicians now routinely offers endovascular treatment (EVT) as a stand-alone therapy instead of or in addition to intravenous thrombolysis for MeVO stroke. ${ }^{4-6}$ Furthermore, 2 randomized trials, ESCAPE-MeVO and DISTAL, are being planned to generate level $1 \mathrm{~A}$ evidence for EVT in MeVO stroke (personal communication: Mayank Goyal, MariosNikos Psychogios, oral communication). The prerequisite for conducting these trials and for appropriate treatment of MeVOs is accurate and reliable $\mathrm{MeVO}$ detection and distinction between MeVOs and large-vessel occlusions (LVOs). However, MeVOs are missed in up to one-third of cases. ${ }^{7}$ Furthermore, distinguishing MeVOs from LVOs is not always straightforward because 
various definitions for the borders between the M1 and M2 segments exist. ${ }^{8}$ The same holds true for the anterior and posterior cerebral arteries. Multiphase CTA (mCTA) is a dynamic imaging method in which an arch-to-vertex CTA is obtained in a manner identical to single-phase CTA. The same contrast bolus is then used to obtain 2 additional series during the peak-venous and late-venous phases, covering only the intracranial vasculature from the skull base to the vertex (Online Supplemental Data). The delayed washout on the second and third phases in the vascular territory downstream to the occlusion may help to improve detection accuracy and interrater agreement for MeVOs.

In this study, we aimed to compare interrater agreement and detection accuracy for MeVOs using single-phase CTA and mCTA.

\section{MATERIALS AND METHODS \\ Patient Sample}

This study was approved by the local institutional review board at the University of Calgary. Data are from the Precise and Rapid Assessment of Collaterals Using Multi-Phase CTA in the Triage of Patients with Acute Ischemic Stroke for IV or IA Therapy (PRove-IT) study (clinicaltrials.gov identifier: NCT02184936). PRove-IT was a prospective multicenter cohort study that enrolled 595 patients who presented with symptoms of acute ischemic stroke. Patients were included if they presented to the emergency department with symptoms consistent with acute ischemic stroke, were older than 18 years of age, and had mCTA and CT perfusion performed within 12 hours of symptom onset and before recanalization therapy. Exclusion criteria were intracranial hemorrhage at baseline NCCT, previous sizeable stroke in the ipsilateral hemisphere, $\mathrm{mRS}>2$ at baseline, estimated creatinine clearance of $<60 \mathrm{~mL} / \mathrm{min}$, contrast material allergy or other contraindications for iodinated contrast, and estimated life expectancy of $<1$ year. ${ }^{9}$ The enrollment period was July 2012 to October 2016. For this study, a sample of 120 patients (20 with no occlusion, 44 with LVO, and 56 with $\mathrm{MeVO}$ in the anterior and posterior circulation) was randomly chosen. The proportions of patients with MeVOs in relation to LVOs and scans without occlusions were chosen so that they approximately reflected the distribution of occlusions in the PRove-IT study.

\section{Imaging}

We exclusively used baseline CTA imaging in this study. The first CTA phase consisted of an arch-to-vertex coverage (CTA head and neck and conventional single-phase CTA). This first phase was used for the single-phase CTA reading session and was followed by skull base-to-vertex coverage for the second (peak venous) and third (late venous) phases of an mCTA acquisition. All 3 phases were available in the mCTA reading session. Detailed mCTA acquisition parameters have been published previously. ${ }^{9}$ Axial images with 1-mm overlap and multiplanar axial, coronal, and sagittal reconstructions with 3-mm thickness, 1-mm intervals, and 1-mm overlap for the first phase were obtained, along with axial minimum intensity projections for all 3 phases with 24-mm thickness and 4-mm intervals, available for the readers.

\section{Image Analysis}

Three blinded readers (a general radiologist with 4 years of experience and 2 neuroradiologists with 7 and 2 years of experience) interpreted the images in 2 separate reading sessions. In the first session, only single-phase CTA (ie, the first CTA phase with arch-to-vertex coverage) was available. In the second session, all 3 mCTA phases were available. Readers were informed that the set of cases included patients with LVO and MeVO in the anterior and posterior circulation as well as cases without any occlusion, but they did not know the distribution of occlusions and occlusion locations. They were blinded to all other baseline imaging, follow-up imaging, and clinical information. Occlusion sites were captured as either no occlusion; intracranial internal carotid artery occlusion; M1, M2, or M3/4 segment MCA occlusion; A1, $\mathrm{A} 2$, or A3/4 segment anterior cerebral artery occlusion; or P1, P2, or P3/4 segment posterior cerebral artery occlusion. Detailed definitions of vessel segments and borders between them as they were used in this study are shown in the Online Supplemental Data. Besides these detailed occlusion sites, occlusions were also grouped into no occlusion versus LVOs (occlusions of the ICA, $\mathrm{M} 1, \mathrm{~A} 1$, or $\mathrm{P} 1$ segments) versus MeVOs (occlusions of the M2/3/ 4 , A2/3/4, or P2/3/4 segments). The reference standard was set by an independent core lab (M.G., interventional neuroradiologist with 24 years of neuroimaging experience), with all imaging information (baseline noncontrast head CT, mCTA, CT perfusion, and follow-up imaging) as well as clinical information being available.

\section{Statistical Analysis}

Interrater agreement for occlusion type (LVO versus $\mathrm{MeVO}$ versus no occlusion) and for detailed occlusion sites was assessed using the Fleiss $\kappa$ for multiple raters ${ }^{10}$ with respective optimismcorrected bootstrapped 95\% confidence intervals. According to common convention, $\kappa<0$ was interpreted as poor agreement; $\kappa=0-0.2$, as slight agreement; $\kappa=0.21-0.40$, as fair agreement; $\kappa=0.41-0.60$, as moderate agreement; $\kappa=0.61-0.80$, as substantial agreement; and $\kappa=0.81-1$, as almost perfect agreement. ${ }^{11}$ The accuracy of MeVO detection overall and for each $\mathrm{MeVO}$ occlusion site was calculated for each reader using classification tables. All analyses were performed in STATA, Version 15.1 (StataCorp).

\section{RESULTS}

A total of 120 cases were included in the study, among them 20 with no visible occlusion, 44 LVOs (22 intracranial ICA occlusions, 2 vertebral artery occlusions, 1 basilar artery occlusion, 18 M1 occlusions, and $1 \mathrm{P} 1$ occlusion), and $56 \mathrm{MeVOs}$ (21 M2 occlusions, 12 M3/4 occlusions, 5 A2 occlusions, 4 A3/4 occlusions, and $14 \mathrm{P} 2$ occlusions).

\section{Interrater Agreement}

Interrater agreement for occlusion type was moderate for singlephase CTA $(\kappa=0.58 ; 95 \%$ CI, $0.56-0.62)$ and significantly improved to excellent agreement when mCTA was used $(\kappa=0.81$; 95\% CI, 0.78-0.83). Interrater agreement for detailed occlusion sites (see the Online Supplemental Data for detailed definitions) was 


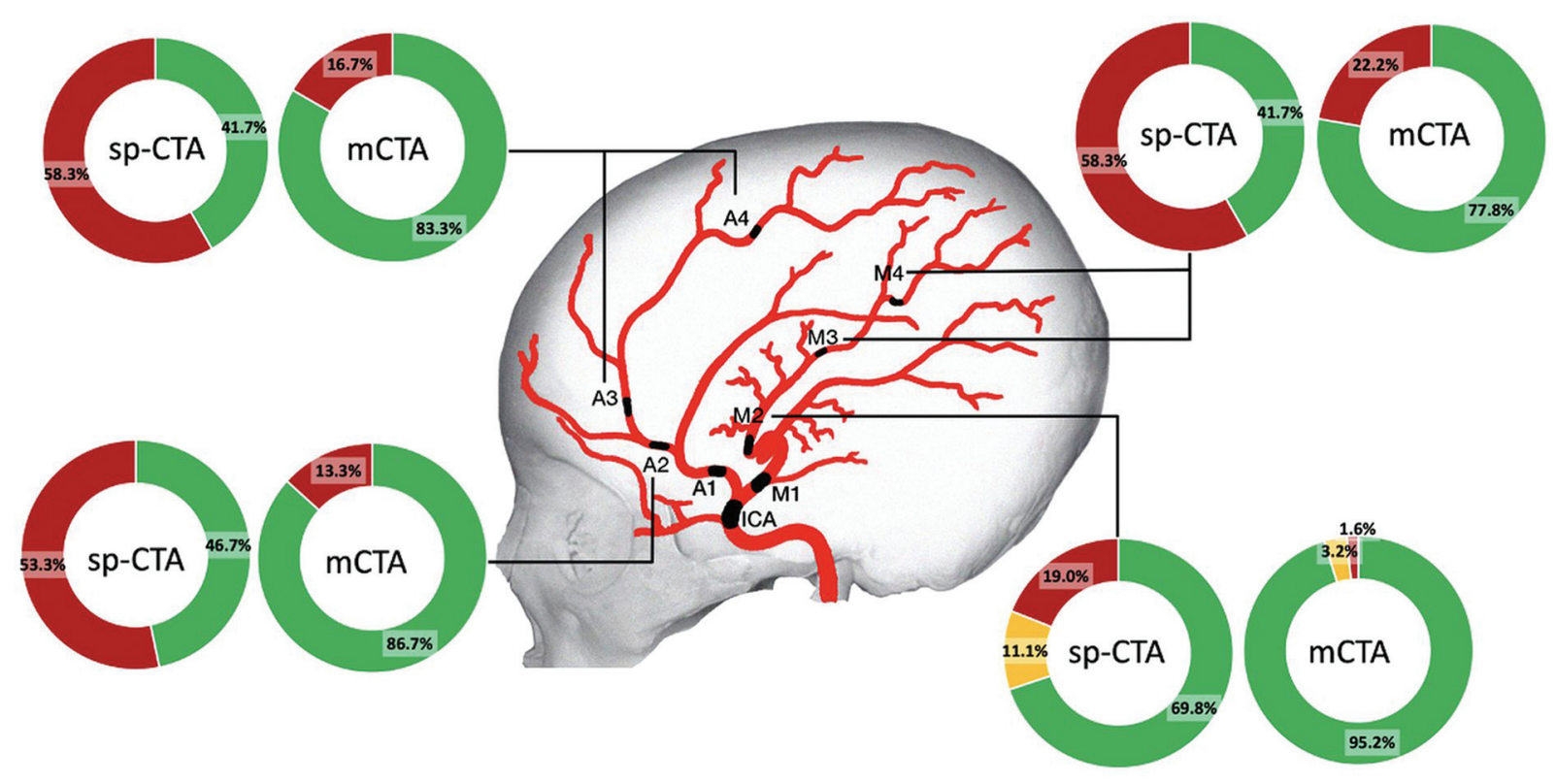

A

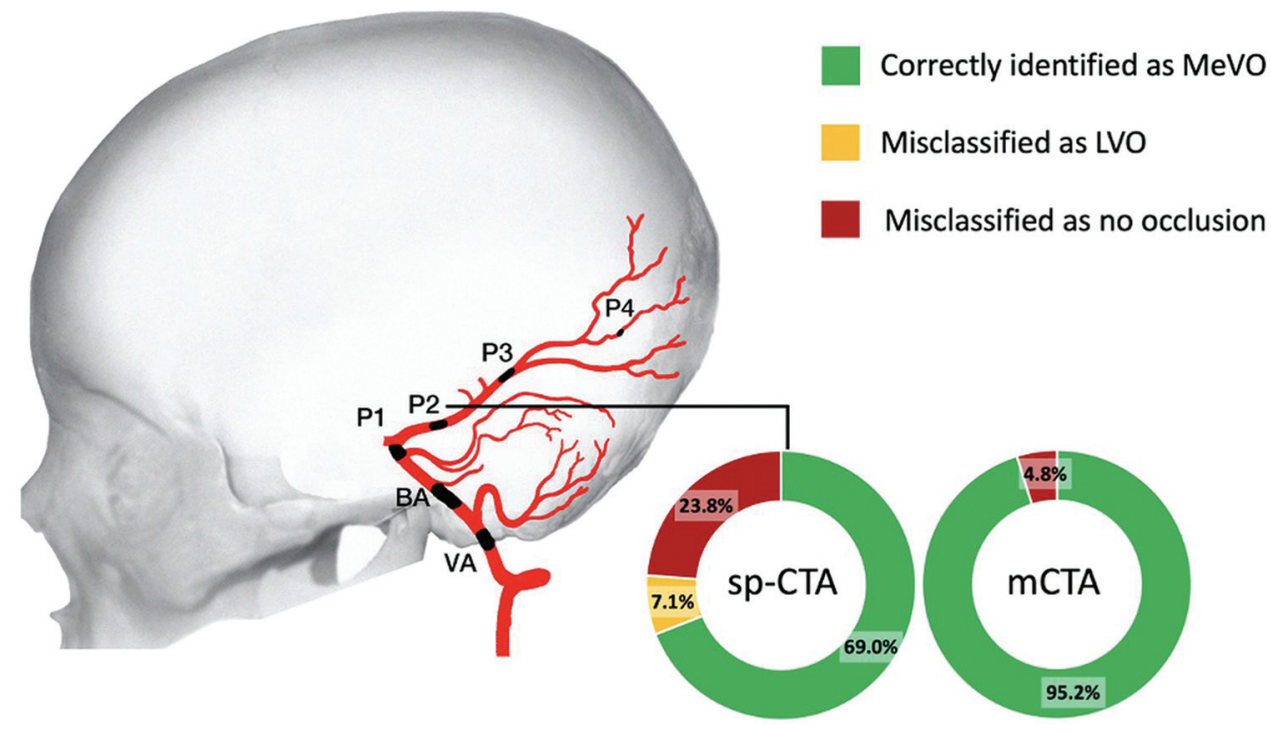

FIGURE. Proportion of MeVOs that were correctly classified as MeVOs (green), misclassified as LVOs (yellow), and misclassified as no occlusion (red) on single-phase and multiphase CTA, stratified per occlusion site. A, Proportions for M2, M3/4, A2, and A3/4 MeVOs. B, Proportions for P2 MeVOs. sp-CTA indicates single-phase CT angiography.

moderate for single-phase CTA $(\kappa=0.55 ; 95 \%$ CI, $0.53-0.56)$ and substantial for mCTA $(\kappa=0.71 ; 95 \%$ CI, $0.67-0.74)$.

\section{Accuracy of MeVO Detection}

Classification tables for the 3 readers on single-phase CTA and mCTA are shown in the Online Supplemental Data. On singlephase CTA, readers 1, 2, and 3 classified 33/56 (59\%), 34/56 (61\%), and 32/56 (57\%) MeVOs correctly as such. Most of the misclassified MeVOs on single-phase CTA (21/23 [91\%], 16/22 [73\%], and 22/24 [92\%]) were erroneously classified as "no occlusion," while only very few were mistaken for LVOs. On mCTA, 48/56 (86\%), 50/56 (89\%), and 50/56 (89\%) MeVOs were classified correctly, and most misclassified MeVOs (6/8 [75\%], 4/6 [67\%], and 5/6 [83\%]) were misclassified as no occlusion. The Figure shows the proportion of MeVOs that were correctly classified as MeVOs, misclassified as LVOs, and misclassified as no occlusion on single-phase and multiphase CTA, stratified per occlusion site.

\section{DISCUSSION}

Interrater agreement for occlusion sites in this study was moderate when using single-phase CTA and almost perfect with mCTA. The accuracy of $\mathrm{MeVO}$ detection for all 3 readers ranged between $57 \%$ and $61 \%$ on single-phase CTA and improved to $86 \%-89 \%$ with mCTA.

In the past, it was thought that $\mathrm{MeVO}$ strokes resulted in relatively favorable clinical outcomes due to their more distal occlusion location and smaller ischemic tissue volume compared with LVOs. However, data from large, prospective cohort studies and a pooled meta-analysis from randomized trials showed that only half of the patients with MeVOs achieve a good functional outcome at 
90 days with current best medical management. ${ }^{12-14}$ Together with the high efficacy and safety of EVT in LVO stroke, this finding has led many physicians to routinely offer EVT for MeVO stroke. ${ }^{4-6}$

To offer the appropriate treatment, it is, however, necessary that MeVOs are quickly and reliably identified on imaging. Missing a $\mathrm{MeVO}$ on baseline imaging can lead to a delayed diagnosis or misdiagnosis, which may result in a patient not receiving intravenous thrombolysis or not getting transferred to a comprehensive stroke center for EVT. It may also cause misclassification of the patient's symptoms as a stroke mimic and, as a consequence, failure to initiate appropriate stroke work-up and secondary stroke-prevention measures. Unfortunately, recent data suggest that this scenario is not an uncommon one: Fasen et $\mathrm{al}^{7}$ retrospectively reviewed 520 single-phase CTA studies of patients with clinically confirmed acute ischemic stroke and found that M2 occlusions, which are arguably the most easily detectable MeVOs, were 5 times more likely to be overlooked compared with LVOs and were missed in up to onethird of cases. Misses were most common when non-neuroradiologists and trainees interpreted the scans. ${ }^{7}$

We have recently commented on the potential value of mCTA in improving MeVO detection. ${ }^{15}$ Indeed, our study showed an excellent interrater agreement and substantially higher detection accuracy for MeVOs with mCTA compared with single-phase CTA, suggesting that mCTA could be a valuable tool for $\mathrm{MeVO}$ detection. Innovative mCTA display formats, such as color-coded time-variant mCTA maps or mCTA tissue-level perfusion maps, may be able to facilitate MeVO detection even further, but they are not widely available yet. ${ }^{15,16}$

Given the complexity in the anatomic definition of MeVOs (for example, there are different ways to define the border between the M1 and M2 segments of the $\mathrm{MCA}^{8}$ ), one may suspect that some MeVOs will be misclassified as LVOs. This is particularly problematic because establishing high-level evidence for MeVO EVT in randomized trials requires an accurate, reliable, and reproducible $\mathrm{MeVO}$ definition as part of the trial inclusion criteria. However, in our study, most misclassified MeVOs were not mistaken for LVOs but rather erroneously classified as no occlusion, suggesting that the problem of distinguishing MeVOs from LVOs is only a minor one and confirming that the MeVO definition that was previously suggested by our group ${ }^{1}$ and has been used in the current study could potentially be used as a randomized trial inclusion criterion. Furthermore, detection of occlusion of any kind will lead to the correct diagnosis of acute ischemic stroke and trigger appropriate treatment with IV thrombolysis and potentially EVT; in other words, mistaking an MeVO as an LVO is not as fatal as missing it altogether.

The added radiation dose of $1.0 \mathrm{mSv}$ for the 2 additional phases that are obtained in multiphase CTA slightly increases the mean effective dose for a CT-based acute ischemic stroke imaging protocol from 7.0 to $8.0 \mathrm{mSv}$, and it is generally deemed acceptable, given the severity of the condition. ${ }^{9}$

\section{Limitations}

This study has limitations. First, there is controversy about the exact anatomic definition of the M1 versus the M2 segment, and the situation is arguably even more complex in the anterior and posterior cerebral arteries, in which anatomy is even more variable ${ }^{17}$ and includes variants such as an azygos anterior cerebral artery and fetal origin of the posterior cerebral artery. Nevertheless, this variability simply reflects the clinical reality, and some disagreement in distinguishing MeVOs versus LVOs is, therefore, expected. Second, we chose to compare single-phase CTA with mCTA because they are closely related in their acquisition technique, have identical contrast doses and comparable radiation doses, and can be performed without any postprocessing software. ${ }^{9}$ We did not include other imaging modalities such as CT perfusion or MR imaging and are, therefore, not able to comment on the value of these imaging modalities for $\mathrm{MeVO}$ assessment. Third, not all MeVO sites were represented in this study; for example, we could not include any cases of P3 occlusion simply because they did not occur in our dataset. Fourth, the readers in this study relied exclusively on single-phase CTA and mCTA to assess occlusion sites and did not have access to any other imaging or clinical information, while in a real-world scenario, the reader will almost always have access to clinical information and baseline noncontrast CT. However, one could argue that access to this information may, if anything, have improved the readers' performance. Last, distinguishing between MeVOs and LVOs, which was part of the current study, while being critically important for randomized MeVO trials, may be only of limited usefulness in clinical practice once the safety and efficacy of MeVO EVT has been proven.

\section{CONCLUSIONS}

Interrater agreement for MeVOs is moderate when using singlephase CTA and almost perfect with mCTA. The accuracy of $\mathrm{MeVO}$ detection is higher with mCTA compared with singlephase CTA, suggesting that mCTA might be a valuable tool that allows reliable diagnosis of $\mathrm{MeVO}$ stroke.

\section{ACKNOWLEDGMENTS}

The authors are most grateful to all enrolling sites.

Disclosure forms provided by the authors are available with the full text and PDF of this article at www.ajnr.org.

\section{REFERENCES}

1. Goyal M, Ospel JM, Menon BK, et al. MeVO: the next frontier? J Neurointerv Surg 2020;12:545-47 CrossRef Medline

2. Duloquin G, Graber M, Garnier L, et al. Incidence of acute ischemic stroke with visible arterial occlusion: a population-based study (Dijon Stroke Registry). Stroke 2020;51:2122-30 CrossRef Medline

3. Saver JL, Chapot R, Agid R, et al; Distal Thrombectomy Summit Group. Thrombectomy for distal, medium vessel occlusions: a consensus statement on present knowledge and promising directions. Stroke 2020;51:2872-84 CrossRef Medline

4. Almekhlafi M, Ospel JM, Saposnik G, et al. Endovascular treatment decisions in patients with M2 segment MCA occlusions. AJNR Am J Neuroradiol 2020;41:280-85 CrossRef Medline

5. Cimflova P, Kappelhof M, Singh N, et al. Factors influencing thrombectomy decision making for primary medium vessel occlusion stroke. J Neurointerv Surg 2021 May 5 [Epub ahead of print] CrossRef Medline 
6. Kappelhof M, Ospel J, Kashani N, et al. Influence of intravenous alteplase on endovascular treatment decision-making in acute ischemic stroke due to primary medium-vessel occlusion: a casebased survey study. J Neurointerv Surg 2021 May 25 [Epub ahead of print] CrossRef Medline

7. Fasen B, Heijboer RJJ, Hulsmans FH, et al. CT angiography in evaluating large-vessel occlusion in acute anterior circulation ischemic stroke: factors associated with diagnostic error in clinical practice. AJNR Am J Neuroradiol 2020;41:607-11 CrossRef Medline

8. Goyal M, Menon BK, Krings T, et al. What constitutes the M1 segment of the middle cerebral artery? J Neurointerv Surg 2016;8:127377 CrossRef Medline

9. Menon BK, d'Esterre CD, Qazi EM, et al. Multiphase CT angiography: a new tool for the imaging triage of patients with acute ischemic stroke. Radiology 2015;275:510-20 CrossRef Medline

10. Fleiss JL, Cohen J. The equivalence of weighted kappa and the intraclass correlation coefficient as measures of reliability. Educational and Psychological Measurement 1973;33:613-19 CrossRef

11. Hartling LH, Milne A. Validity and inter-rater reliability testing of quality assessment instruments. https://www.ncbi.nlm.nih.gov/ books/NBK92295/table/methods.t2/. Accessed August 9, 2021
12. Lima FO, Furie KL, Silva GS, et al. Prognosis of untreated strokes due to anterior circulation proximal intracranial arterial occlusions detected by use of computed tomography angiography. JAMA Neurol 2014;71:151-57 CrossRef Medline

13. Menon BK, Hill MD, Davalos A, et al. Efficacy of endovascular thrombectomy in patients with M2 segment middle cerebral artery occlusions: meta-analysis of data from the HERMES Collaboration. J Neurointerv Surg 2019;11:1065-69 CrossRef Medline

14. Ospel JM, Menon BK, Demchuk AM, et al. Clinical course of acute ischemic stroke due to medium vessel occlusion with and without intravenous alteplase treatment. Stroke 2020;51:3232-40 CrossRef Medline

15. Ospel JM, Qiu W, Goyal M. Missed medium-vessel occlusions on CT angiography: make it easier ... easily! AJNR Am J Neuroradiol 2020;41:E73-74 CrossRef Medline

16. Ospel JM, Volny O, Qiu W, et al. Displaying multiphase CT angiography using a time-variant color map: practical considerations and potential applications in patients with acute stroke. AJNR Am J Neuroradiol 2020;41:200-05 CrossRef Medline

17. van der Zwan A, Hillen B, Tulleken CA, et al. Variability of the terrritories of the major cerebral arteries. J Neurosurg 1992;77:927-40 CrossRef Medline 